\title{
Correlation femtoscopy
}

\author{
R. Lednickýa b* \\ a Joint Institute for Nuclear Research, \\ Dubna, Moscow Region, 141980, Russia \\ ${ }^{\mathrm{b}}$ Institute of Physics ASCR, \\ Na Slovance 2, 18221 Prague 8, Czech Republic
}

The basics of correlation femtoscopy, recent results from femtoscopy in relativistic heavy ion collisions and their consequences are shortly reviewed.

\section{Introduction}

The momentum correlations of two or more particles at small relative momenta in their center-of-mass (c.m.) system are widely used to study space-time characteristics of the production processes on a level of $\mathrm{fm}=10^{-15} \mathrm{~m}$, so serving as a correlation femtoscopy tool. Particularly, for non-interacting identical particles, like photons or, to some extent, pions, these correlations result from the interference of the production amplitudes due to the symmetrization requirement of quantum statistics (QS) [12345].

There exists [2345678 an analogy of the momentum QS correlations of photons with the space-time correlations of the intensities of classical electromagnetic fields used in astronomy to measure the angular radii of stellar objects based on the superposition principle (HBT effect) [9]. This analogy is sometimes misunderstood and the momentum correlations are mixed up with the space-time (HBT) correlations in spite that their orthogonal character and thus the absence of the former in astronomy measurements due to extremely large space-time extent of stellar objects (and vice versa) was already pointed out in early paper [8]. Note that though the space-time (HBT) correlations are absent in the subatomic measurements, they can still be used in laboratory as an intensity-correlation spectroscopy tool (see [1011] and references therein).

The momentum QS correlations were first observed as an enhanced production of the pairs of identical pions with small opening angles (GGLP effect [1]). Later on, Kopylov and Podgoretsky settled the basics of correlation femtoscopy in more than 20 papers (see a review [5]) and developed it as a practical tool; particularly, they suggested to study the interference effect in terms of the correlation function, proposed the mixing techniques to construct the uncorrelated reference sample and clarified the role of the space-time characteristics of particle production in various physical situations.

\footnotetext{
*Work supported by the Grant Agency of the Czech Republic under contract 202/04/0793 and partly carried out within the scope of the GDRE: Heavy ions at ultrarelativistic energies - a European Research Group comprising IN2P3/CNRS, EMN, University of Nantes, Warsaw University of Technology, JINR Dubna, ITEP Moscow and BITP Kiev.
} 
The momentum correlations of particles emitted at nuclear distances are also influenced by the effect of final state interaction (FSI) [1213141516]. Thus the effect of the Coulomb interaction dominates the correlations of charged particles at very small relative momenta (of the order of the inverse Bohr radius $|a|^{-1}$ of the two-particle system), respectively suppressing or enhancing the production of particles with like or unlike charges. Though the FSI effect complicates the correlation analysis, it is an important source of information allowing for the coalescence femtoscopy (see, e.g., 17181920]), the correlation femtoscopy with unlike particles [141516] including the access to the relative space-time asymmetries in particle production [212223242526272829303132] and a study of strong interactions between specific particles [283132].

In this review, I will concentrate on the assumptions behind the correlation femtoscopy formalism and discuss the recent results obtained from the femtoscopy analysis of like and unlike particle correlations in relativistic heavy ion collisions. One can inspect recent reviews 32 33 34] for a number of important topics that are not touched here, such as non-Gaussian tails, imaging techniques, correlations of penetrating probes, comparison of different colliding systems or spin correlations.

\section{Assumptions}

The two-particle correlation function $\mathcal{R}\left(p_{1}, p_{2}\right)$ is usually defined as a ratio of the measured two-particle distribution to the reference one obtained by mixing particles from different events of a given class, normalized to unity at sufficiently large relative momenta. The space-time information contained in the momentum correlations is usually extracted based on the following assumptions:

(i) The mean freeze-out phase space density $\langle f\rangle$ is assumed sufficiently small so that only the mutual QS and FSI effects can be considered when calculating the correlation function of two particles emitted with a small relative momentum $Q=2 k^{*}$ in their c.m. system. This two-particle approximation may not be justified for the rare pairs associated with large phase-space density fluctuations and also in low energy heavy ion reactions when the particles are produced in a mean field of residual nuclei. To deal with this field a quantum adiabatic (factorisation) approach 3536 or the transport simulations (see, e.g., 373839]) can be used. At high energies, there are also some attempts to account for the mean field effects in eikonal 404142] and optical potential 4344] approaches, the latter giving some tens of percent change of the outgoing phase space density profiles in heavy ion collisions at RHIC assuming however unrealistic in-medium masses at the kinetic freeze-out and neglecting a rapid time dependence of the potential. As for $\langle f\rangle$, it increases with energy and for central lead-lead or gold-gold collisions seems to saturate at the highest SPS energy [33 45] (see, however, 46]). The saturated $\langle f\rangle$ is substantially smaller than unity for pions with $p_{t}>0.2 \mathrm{Gev} / c$ so pointing to negligible multiboson effects (see, e.g., 4748) in this $p_{t}$-region.

(ii) The momentum dependence of the one-particle emission probabilities is assumed inessential when varying the particle four-momenta $p_{1}$ and $p_{2}$ by the amount characteristic for the correlations due to QS and FSI. This smoothness assumption, requiring the components of the mean space-time distance between particle emitters much larger than those of the space-time extent of the emitters, is well justified for heavy ion collisions. 
(iii) An independent or incoherent particle emission is assumed. This assumption is quite reasonable for a dominant part of particle pairs produced in heavy ion collisions and is consistent with the observed strength of two- and three-pion correlation functions (see, e.g., 495051 ).

(iv) To simplify the calculation of the FSI effect, the Bethe-Salpeter amplitude describing two particles emitted at space-time points $x_{i}=\left\{t_{i}, \mathbf{r}_{i}\right\}$ and detected with fourmomenta $p_{i}$ is usually calculated at equal emission times in the pair c.m. system; i.e. the reduced non-symmetrized Bethe-Salpeter amplitude (with the removed unimportant phase factor due to the c.m. motion), depending only on the relative four-coordinate $\Delta x \equiv x_{1}-x_{2}=\{t, \mathbf{r}\}$ and the generalized relative momentum $\widetilde{q}=q-P(q P) / P^{2}$ $\left(q=p_{1}-p_{2}, P=p_{1}+p_{2}\right.$ and $q P=m_{1}^{2}-m_{2}^{2}$; in the two-particle c.m. system, $\mathbf{P}=0$, $\tilde{q}=\left\{0,2 \mathbf{k}^{*}\right\}$ and $\left.\Delta x=\left\{t^{*}, \mathbf{r}^{*}\right\}\right)$, is substituted by a stationary solution $\psi_{-\mathbf{k}^{*}}^{S(+)}\left(\mathbf{r}^{*}\right)$ of the scattering problem having at large distances $r^{*}$ the asymptotic form of a superposition of the plane and outgoing spherical waves (the minus sign of the vector $\mathbf{k}^{*}$ corresponds to the reverse in time direction of the emission process). This equal time approximation is valid on conditions [14,52] $\left|t^{*}\right| \ll m_{2,1} r^{* 2}$ for $\operatorname{sign}\left(t^{*}\right)= \pm 1$ respectively. These conditions are usually satisfied for heavy particles like kaons or nucleons. But even for pions, the $t^{*}=0$ approximation merely leads to a slight overestimation (typically $<5 \%$ ) of the strong FSI effect 52 and, it doesn't influence the leading zero-distance $\left(r^{*} \ll|a|\right)$ effect of the Coulomb FSI.

Then, for non-identical particles,

$\mathcal{R}\left(p_{1}, p_{2}\right) \doteq \sum_{S} \tilde{\rho}_{S}\left\langle\left|\psi_{-\mathbf{k}^{*}}^{S(+)}\left(\mathbf{r}^{*}\right)\right|^{2}\right\rangle_{S}$

similar to the Fermi factor taking into account the Coulomb FSI in $\beta$-decay. For identical particles, the amplitude in Eq. (11) enters in a symmetrized form:

$\psi_{-\mathbf{k}^{*}}^{S(+)}\left(\mathbf{r}^{*}\right) \rightarrow\left[\psi_{-\mathbf{k}^{*}}^{S(+)}\left(\mathbf{r}^{*}\right)+(-1)^{S} \psi_{\mathbf{k}^{*}}^{S(+)}\left(\mathbf{r}^{*}\right)\right] / \sqrt{2}$

The averaging in Eq. (11) is done over the four-coordinates of the emitters at a given total spin $S$ of the two-particles, $\widetilde{\rho}_{S}$ is the corresponding population probability, $\sum_{S} \widetilde{\rho}_{S}=1$. For unpolarized particles with spins $s_{1}$ and $s_{2}$ the probability $\widetilde{\rho}_{S}=(2 S+1) /\left[\left(2 s_{1}+1\right)\left(2 s_{2}+1\right)\right]$. Note that in the case of small $k^{*}$, we are interested in, the short-range interaction is dominated by central forces and s-waves so that, neglecting a weak spin dependence of the Coulomb interaction, the spin dependence of the two-particle amplitude enters only through the total spin $S$.

(v) The two-particle approximation in (i) and FSI separation in the Bethe-Salpeter amplitudes of the elastic transitions $1+2 \rightarrow 1+2$ implies a long FSI time as compared with the characteristic production time, i.e. the channel momentum $k^{*}$ much less than typical production momentum transfer of hundreds $\mathrm{MeV} / c$. In fact, the long-time FSI can be separated also in the inelastic transitions, $1+2 \rightarrow 3+4$, characterized by a slow relative motion in both entrance and exit channels [5352. The necessary condition is an approximate equality of the sums of particle masses in the channels $\alpha=1+2$ and $\beta=3+4$. In the presence of such transitions the amplitudes in Eqs. (11) and (2) should be substituted by the solutions of the two-channel scattering problem, i.e., formally, $S \rightarrow S, \alpha \alpha$ and $S, \beta \alpha$. In practice, the particles 1,3 and 2, 4 are members of the same 
isomultiplets (as, e.g., in the transition $\pi^{-} p \leftrightarrow \pi^{0} n$ ) so that one can put $\widetilde{\rho}_{S, \alpha \alpha}=\widetilde{\rho}_{S, \beta \alpha}$ and assume the same $\mathbf{r}^{*}$-distributions in the channels $\alpha$ and $\beta$.

\section{Femtoscopy with identical particles}

For identical pions or kaons, the effect of the strong FSI is usually small and the effect of the Coulomb FSI can be in first approximation simply corrected for (see [54 and references therein). The corrected correlation function is determined by the QS symmetrization only (see Eq. (2) and substitute the non-symmetrized amplitude by the plane wave $\mathrm{e}^{i q x / 2}$ ):

$\mathcal{R}\left(p_{1}, p_{2}\right)=1+\langle\cos (q \Delta x)\rangle$.

Its characteristic feature is the presence of the interference maximum at small components of the relative four-momentum $q$ with the width reflecting the inverse space-time extent of the effective production region.

Note that the on-shell constraint $q_{0}=\mathbf{v q}\left(\mathbf{v}=\mathbf{P} / P_{0}\right.$ is the pair velocity) makes the $q$-dependence of the correlation function essentially three-dimensional [4] (particularly, in pair c.m. system, $q \Delta x=-2 \mathbf{k}^{*} \mathbf{r}^{*}$ ) and thus makes impossible the unique Fourier reconstruction of the space-time characteristics of the emission process. However, within realistic models, the directional and velocity dependence of the correlation function can be used to determine both the duration of the emission and the form of the emission region [2345], as well as - to reveal the details of the production dynamics (such as collective flows; see, e.g., [55 5657] and reviews [5859]). For this, the correlation functions can be analyzed in terms of the out $(\mathrm{x})$, side $(\mathrm{y})$ and longitudinal $(\mathrm{z})$ components of the relative momentum vector $\mathbf{q}=\left\{q_{x}, q_{y}, q_{z}\right\}$ 606162 63]; the out and side denote the transverse components of the vector $\mathbf{q}$, the out direction is parallel to the transverse component of the pair three-momentum. The corresponding correlation widths are usually parameterized in terms of the Gaussian correlation radii $R_{i}$,

$\mathcal{R}\left(p_{1}, p_{2}\right)=1+\lambda \exp \left(-R_{x}^{2} q_{x}^{2}-R_{y}^{2} q_{y}^{2}-R_{z}^{2} q_{z}^{2}-2 R_{x z}^{2} q_{x} q_{z}\right)$

and their dependence on pair rapidity and transverse momentum is studied. The correlation strength parameter $\lambda$ represents a fraction of the pairs of identical pions or kaons emitted by independent short-lived sources. The form of Eq. (44) assumes azimuthal symmetry of the production process [5860]. Generally, e.g., in case of the correlation analysis with respect to the reaction plane, all three cross terms $q_{i} q_{j}$ contribute [64].

It is well known that particle correlations at high energies usually measure only a small part of the space-time emission volume, being only slightly sensitive to its increase related to the fast longitudinal motion of particle sources. In fact, due to limited source decay momenta of few hundred $\mathrm{MeV} / c$, the correlated particles with nearby velocities are emitted by almost comoving sources and so - at nearby space-time points. The dynamical examples are sources-resonances, colour strings or hydrodynamic expansion. To substantially eliminate the effect of the longitudinal motion, the correlations can be analyzed in terms of the invariant variable $Q=\left(-\widetilde{q}^{2}\right)^{1 / 2}=2 k^{*}$ and the components of the momentum difference in pair c.m. system $\left(\mathbf{q}^{*} \equiv \mathbf{Q}=2 \mathbf{k}^{*}\right)$ or in the longitudinally comoving system (LCMS) 65]. In LCMS each pair is emitted transverse to the reaction axis so that the generalized relative momentum $\widetilde{\mathbf{q}}$ coincides with $\mathbf{q}^{*}$ except for the component $\widetilde{q}_{x}=\gamma_{t} q_{x}^{*}$, where $\gamma_{t}$ is the LCMS Lorentz factor of the pair. 
Particularly, in the case of one-dimensional boost invariant expansion, the longitudinal correlation radius in the LCMS reads [57] $R_{z} \approx\left(T / m_{t}\right)^{1 / 2} \tau$, where $T$ is the freeze-out temperature, $\tau$ is the proper freeze-out time and $m_{t}$ is the transverse particle mass. In this model, the side radius measures the transverse radius of the system while the square of the out radius gets an additional contribution $\left(p_{t} / m_{t}\right)^{2} \Delta \tau^{2}$ due to the finite emission duration $\Delta \tau$. The additional transverse expansion leads to a slight modification of the $p_{t}$-dependence of the longitudinal radius and - to a noticeable decrease of the side radius and the spatial part of the out radius with $p_{t}$. Since the freeze-out temperature and the transverse flow determine also the shapes of the $m_{t}$-spectra, the simultaneous analysis of correlations and single particle spectra for various particle species allows to disentangle all the freeze-out characteristics (see a review [58]). It appears that with the increasing energy of heavy ion collisions from AGS and SPS up to the highest energies at RHIC, the correlation data show rather weak energy dependence 66 67] and point to the kinetic freeze-out temperature somewhat below the pion mass, a strong transverse flow (with the mean transverse flow velocity at RHIC exceeding half the velocity of light), a short evolution time of 8-10 fm/c and a very short emission duration of about 2-3 fm/c (see, e.g., a recent review [33]). The short evolution and emission duration at RHIC are also supported by the correlation analysis with respect to the reaction plane 6768 .

The small time scales at RHIC were not expected in traditional transport and hydrodynamic models (see, e.g., 6970]) and may indicate an explosive character of particle production [7172] or, large parton cross sections [73] and early hadronization [74] as in the corresponding successful multi-phase and hadron transport models. In view of a successful hydrodynamical description of the elliptic flow at RHIC (see, however, 75]) the failure of hydrodynamics to explain the femtoscopy data is often considered as a puzzle. However, it is still possible that the overestimation of the outward and longitudinal radii will be cured in the full three-dimensional hydrodynamic calculations with the modified initial conditions (earlier thermalization time or initial transverse flow 7677 ), tuned equation of state with the account for chemical non-equilibrium after hadronization [6777879] and a better modeling of the freeze-out process (including continuous emission during the hydrodynamic stage [8081] and coupling to the hadron cascade 8283]). This hope is supported by a better description of the correlation radii in a recent three-dimensional hydrodynamic model [84] and by a successful description of the SPS and RHIC data in a number of hydro-motivated parametrizations (see a brief review [85]). The success of these simple parametrizations can be caused by the formation of particle spectra and correlation radii during an early stage of the kinetic freeze-out (see, e.g., [837374]). It was argued [81] that such an early spectra formation can be related with the fact that the solution of the non-relativistic Boltzmann equation at spherically symmetric initial conditions exactly coincides with free streaming (see, e.g., [86]). Indeed, numerical solutions of relativistic Boltzmann equation at anisotropic initial conditions showed only a slight deviation from free streaming even in the case of a large number of rescatterings [87.

\section{Femtoscopy with unlike particles}

The complicated dynamics of particle production, including resonance decays and particle rescatterings, leads to essentially non-Gaussian tail of the $r^{*}$-distribution. Therefore, 
due to different $r^{*}$-sensitivity of the QS, strong and Coulomb FSI effects, one has to be careful when analyzing the correlation functions in terms of simple models. Thus, the QS and strong FSI effects are influenced by the $r^{*}$-tail mainly through the correlation strength parameter $\lambda$ while, the shape of the Coulomb FSI is sensitive to the distances as large as the pair Bohr radius $|a|$ (hundreds fm for the pairs containing pions). These problems can be at least partially overcome with the help of transport code simulations accounting for the dynamical evolution of the emission process and providing the phase space information required to calculate the QS and FSI effects on the correlation function.

Thus, in a preliminary analysis of the NA49 correlation data from central $\mathrm{Pb}+\mathrm{Pb}$ 158 AGeV collisions [28, the transport RQMD v.2.3 code was used. To account for a possible mismatch in $\left\langle r^{*}\right\rangle$, the correlation functions were calculated with the space-time coordinates of the emission points scaled by $0.7,0.8$ and 1 . The scale parameter was then fitted using the quadratic interpolation. The fits of the $\pi^{+} \pi^{-}, \pi^{+} p$ and $\pi^{-} p$ correlation function indicate that RQMD overestimates the distances $r^{*}$ by $10-20 \%$ thus indicating an underestimation of the collective flow in this model.

Recently, there appeared data on $p \Lambda$ correlation functions from $A u+A u$ experiments E985 at AGS [88, STAR at RHIC 31] and $P b+P b$ experiment NA49 at SPS CERN 29]. As the Coulomb FSI is absent in $p \Lambda$ system, one avoids here the problem of its sensitivity to the $r^{*}$-tail. Also, the absence of the Coulomb suppression of small relative momenta makes this system more sensitive to the radius parameters as compared with $p p$ correlations [89]. In spite of rather large statistical errors, a significant enhancement is seen at low relative momentum, consistent with the known singlet and triplet $p \Lambda \mathrm{s}$-wave scattering lengths. In fact, the fits using the analytical expression for the correlation function [14] yield the correlation radii of 3-4 fm in agreement with the radii obtained from $p p$ correlations in the same experiments. These radii are smaller than those obtained from two-pion and two-kaon correlation functions at the same transverse momenta and are in qualitative agreement with the approximate $m_{t}$ scaling expected in the case of the collective expansion.

\section{Accessing strong interaction}

In case of a poor knowledge of the strong interaction, which is the case for mesonmeson, meson-hyperon or hyperon-hyperon systems, it can be improved with the help of correlation measurements.

In heavy ion collisions, the effective radius $r_{0}$ of the emission region can be considered much larger than the range of the strong interaction potential. The FSI contribution to the correlation function is then independent of the actual potential form [1490]. At small $Q=2 k^{*}$, it is determined by the s-wave scattering amplitudes $f^{S}\left(k^{*}\right)$ at a given total spin $S$ [14. In case of $\left|f^{S}\right|>r_{0}$, this contribution is of the order of $\left|f^{S} / r_{0}\right|^{2}$ and dominates over the effect of QS. In the opposite case, the sensitivity of the correlation function to the scattering amplitude is determined by the linear term $f^{S} / r_{0}$.

The possibility of the correlation measurement of the scattering amplitudes has been demonstrated [28] in a preliminary analysis of the NA49 $\pi^{+} \pi^{-}$correlation data within the RQMD model. The fitted strong interaction scale, redefining the original s-wave scattering length of $0.23 \mathrm{fm}$, appeared to be significantly lower than unity: $0.63 \pm 0.08$. 
To a similar shift $(\sim 20 \%)$ point also the recent BNL data on $K_{l 4}$ decays [91]. These results are in agreement with the two-loop calculation in the chiral perturbation theory with a standard value of the quark condensate [92].

Recently, also the singlet $\Lambda \Lambda \mathrm{s}$-wave scattering length has been estimated [28 29] based on the fits of the NA49 data from $\mathrm{Pb}+\mathrm{Pb}$ collisions at $158 \mathrm{AGeV}$. Though the fit results are not very restrictive, they likely exclude the possibility of a large singlet scattering length comparable to that of $\sim 20 \mathrm{fm}$ for the two-nucleon system.

The STAR experiment measured for the first time the $p \bar{\Lambda}$ and $\bar{p} \Lambda$ correlation functions and performed simultaneous fit of the correlation radius and the spin-averaged s-wave scattering length. The fitted imaginary part of the scattering length of $\sim 1 \mathrm{fm}$ is in agreement with the $\bar{p} p$ results (thus pointing to about the same $\bar{p} \Lambda$ and $\bar{p} p$ annihilation cross sections) while the real part appears to be more negative 31].

\section{Accessing relative space-time asymmetries}

The correlation function of two non-identical particles, compared with the identical ones, contains a principally new piece of information on the relative space-time asymmetries in particle emission [21]. Since this information enters in the two-particle amplitude $\psi_{-\mathbf{k}^{*}}^{S(+)}\left(\mathbf{r}^{*}\right)$ through the terms odd in $\mathbf{k}^{*} \mathbf{r}^{*} \equiv \mathbf{p}_{1}^{*}\left(\mathbf{r}_{1}^{*}-\mathbf{r}_{2}^{*}\right)$, it can be accessed studying the correlation functions $\mathcal{R}_{+i}$ and $\mathcal{R}_{-i}$ with positive and negative projection $k_{i}^{*}$ on a given direction $\mathbf{i}$ or, - the ratio $\mathcal{R}_{+i} / \mathcal{R}_{-i}$. For example, $\mathbf{i}$ can be the direction of the pair velocity or, any of the out (x), side (y), longitudinal (z) directions. In LCMS, $r_{i}^{*}=r_{i}$ except for $r_{x}^{*} \equiv \Delta x^{*}=\gamma_{t}\left(\Delta x-v_{t} \Delta t\right)$, where $\gamma_{t}$ and $v_{t}$ are the pair LCMS Lorentz factor and velocity. One may see that the asymmetry in the out $(\mathrm{x})$ direction depends on both space and time asymmetries $\langle\Delta x\rangle$ and $\langle\Delta t\rangle$. In case of a dominant Coulomb FSI, the intercept of the correlation function ratio is directly related with the asymmetry $\left\langle r_{i}^{*}\right\rangle$ scaled by the Bohr radius $a=\left(\mu z_{1} z_{2} e^{2}\right)^{-1}: \mathcal{R}_{+i} / \mathcal{R}_{-i} \approx 1+2\left\langle r_{i}^{*}\right\rangle / a$.

A review of the simulation studies of the method sensitivity and the experimental results can be found elsewhere [32]. Here we only note that the out correlation asymmetries between pions, kaons and protons observed in heavy ion collisions at CERN SPS and BNL RHIC are in agreement with practically charge independent meson production and a negative $\langle\Delta x\rangle$ and/or positive $c\langle\Delta t\rangle$ on the level of several fm (assuming $m_{1}<m_{2}$ ) [28 29303132. In fact they are in quantitative agreement with the RQMD transport model as well as with the hydro-motivated blast wave parametrization, both predicting the dominance of the spatial part of the asymmetries generated by large transverse flows. Particularly, the RQMD simulation of central $\mathrm{Pb}+\mathrm{Pb}$ collisions in conditions of the experiment NA49 at $158 \mathrm{AGeV}$ yields practically zero asymmetries for $\pi^{+} \pi^{-}$system while, for $\pi^{ \pm} p$ systems, $\langle\Delta x\rangle \doteq-5.2 \mathrm{fm},\langle\Delta t\rangle \doteq 2.9 \mathrm{fm} / c,\left\langle\Delta x^{*}\right\rangle \doteq-8.5 \mathrm{fm}$. Besides, it predicts $\langle x\rangle$ increasing with particle $p_{t}$ or $v_{t}=p_{t} / m_{t}$, starting from zero due to kinematic reasons. The asymmetry arises because of a faster increase with $v_{t}$ for heavier particle. In fact, the hierarchy $\left\langle x_{\pi}\right\rangle\left\langle\left\langle x_{K}\right\rangle\left\langle\left\langle x_{p}\right\rangle\right.\right.$ is a signal of a universal transversal collective flow [28; one should simply take into account that the mean thermal velocity is smaller for heavier particle and thus washes out the positive shift due to the flow to a lesser extent.

These conclusions can be expressed in a simple analytical form using a hydro-motivated parametrization. Thus, assuming the longitudinal-boost invariance, a linear non-relativistic 
transversal flow velocity profile $\beta_{F}=\beta_{0} r_{t} / r_{0}$, the local thermal momentum distribution characterized by the kinetic freeze-out temperature $T$ and the Gaussian density profile $\exp \left(-r_{t}^{2} /\left(2 r_{0}^{2}\right)\right)$, one confirms a faster rise of $\langle x\rangle$ with $v_{t}$ for heavier particles [32 93]:

$\langle x\rangle=r_{0} v_{t} \beta_{0} /\left(\beta_{0}^{2}+T / m_{t}\right)$.

The size of the shift $\langle x\rangle$ and its mass dependence are weakened when introducing the transverse temperature gradient $\langle\Delta T / T\rangle_{r}$ like in the Buda-Lund model (this gradient just adds to the denominator in Eq. (5)) 94. The observation of large correlation asymmetries at RHIC may thus point against the existence of a hot emitting central zone and the corresponding gradient $\langle\Delta T / T\rangle_{r} \sim 1$ indicated by the analysis of RHIC data within the Buda-Lund model [95. Further quantitative studies are needed to clarify this question.

\section{Conclusions}

Wealth of data on momentum correlations of various particle species $\left(\pi^{ \pm}, K^{ \pm 0}, p^{ \pm}, \Lambda, \Xi\right)$ is available and gives unique space-time information on production characteristics including collective flows. Rather direct evidence for a strong transverse flow in heavy ion collisions at SPS and RHIC is coming from unlike particle correlation asymmetries. Being sensitive to relative time delays and collective flows, the correlation asymmetries can be especially useful to study the effects of phase transitions. Weak energy dependence of correlation radii contradicts to traditional hydrodynamic calculations which overestimate longitudinal and, especially, outward radii at RHIC. It remains to be clarified whether this can be cured in full three-dimensional hydrodynamic calculations with a more refined treatment of initial conditions, equation of state and freeze-out process. A number of succesful hydro motivated parametrizations give useful hints in this direction. The momentum correlations between specific particles yield also a valuable information on the strong interaction hardly accessible by other means.

\section{REFERENCES}

1. G. Goldhaber et al., Phys. Rev. 120 (1960) 300.

2. V.G. Grishin, G.I. Kopylov and M.I. Podgoretsky, Sov. J. Nucl. Phys. 13 (1971) 638.

3. G.I. Kopylov and M.I. Podgoretsky, Sov. J. Nucl. Phys. 15 (1972) 219.

4. G.I. Kopylov, Phys. Lett. 50B (1974) 472.

5. M.I. Podgoretsky, Sov. J. Part. Nucl. 20 (1989) 266.

6. E.V. Shuryak, Phys. Lett. 44B (1973) 387.

7. G. Cocconi, Phys. Lett. 49B (1974) 459.

8. G.I.Kopylov and M.I.Podgoretsky, Sov. Physics JETP 42 (1975) 211.

9. R. Hanbury-Brown and R.Q. Twiss, Nature 178 (1956) 1046.

10. M.L. Goldberger, H.W. Lewis and K.M. Watson, Phys. Rev. 142 (1966) 25.

11. D.T. Phillips, H. Kleiman and S.P. Davis, Phys. Rev. 153 (1967) 113.

12. S.E. Koonin, Phys. Lett. 70B (1977) 43.

13. M. Gyulassy, S.K. Kauffmann and L.W. Wilson, Phys. Rev. C 20 (1979) 2267.

14. R. Lednicky and V.L. Lyuboshitz, Sov. J. Nucl. Phys. 35 (1982) 770; Proc. CORINNE 90, Nantes, France, 1990 (ed. D. Ardouin, World Sci., 1990) p. 42. 
15. D.H. Boal and J.C. Shillcock, Phys. Rev. C 33 (1986) 549.

16. D.H. Boal, C.-K. Gelbke and B.K. Jennings, Rev. Mod. Phys. 62 (1990) 553.

17. H. Sato and K. Yazaki, Phys. Lett. 98B (1981) 153.

18. V.L. Lyuboshitz, Sov. J. Nucl. Phys. 48 (1988) 956.

19. S. Mrowczynski, Phys. Lett. 277B (1992) 43; ibid. 308B (1993) 216.

20. R. Scheibl and U. Heinz, Phys. Rev. C 59 (1999) 1585.

21. R. Lednicky et al., Phys. Lett. 373B (1996) 30.

22. B. Erazmus et al., CERN Note ALICE-INT-1995-43.

23. S. Voloshin et al., Phys. Rev. Lett. 79 (1997) 4766.

24. D. Ardouin et al., Phys. Lett. 446B (1999) 191.

25. R. Lednicky, S. Panitkin and N. Xu, arXiv:nucl-th/0304062.

26. R. Lednicky, arXiv:nucl-th/0304063, 0304064.

27. D. Miskowiec et al. (E877), arXiv:nucl-ex/9808003.

28. R. Lednicky, NA49 Note number 210 (1999); arXiv:nucl-th/0112011, 0212089.

29. Ch. Blume (NA49), Nucl. Phys. A 715 (2003) 55c.

30. J. Adams et al. (STAR), Phys. Rev. Lett. 91 (2003) 262302.

31. A. Kisiel (STAR), J. Phys. G 30 (2004) S1059.

32. R. Lednicky, arXiv:nucl-th/0305027; Phys. Atom. Nucl. 67 (2004) 72.

33. M. Lisa et al., arXiv:nucl-ex/0505014.

34. T. Csörgo", arXiv:nucl-th/0505019.

35. L. Martin et al., Nucl. Phys. A 604 (1996) 69.

36. H.W. Barz, Phys. Rev. C 59 (1999) 2214; ibid. 53 (1996) 2536.

37. H. Sorge et al., Phys. Lett. 243B (1990) 7.

38. W.G. Gong et al., Phys. Rev. C 43 (1991) 781.

39. M. Isse et al., arXiv:nucl-th/0502058.

40. C.Y. Wong, J. Phys. G 30 (2004) S1053.

41. W.N. Zhang et al., Chin. Phys. Lett. 21 (2004) 1918.

42. J.I. Kapusta and Y. Li, arXiv:nucl-th/0503075.

43. J.G. Cramer et al., Phys. Rev. Lett. 94 (2005) 102302.

44. S. Pratt, arXiv:nucl-th/0508029.

45. S.V. Akkelin and Yu.M. Sinyukov, arXiv:nucl-th/0505045.

46. D. Ferenc et al., Phys. Lett. 457B (1999) 347.

47. R. Lednicky et al., Phys. Rev. C 61 (2000) 034901.

48. U. Heinz, P. Scotto and Q.H. Zhang, Annals Phys. 288 (2001) 325.

49. C. Adams et al. (STAR), Phys. Rev. Lett. 91 (2003) 262301.

50. K. Morita, S. Muroya and H. Nakamura, arXiv:nucl-th/0310057.

51. M. Csanád (PHENIX), arXiv:nucl-ex/0509042, these proceedings.

52. R. Lednicky, arXiv:nucl-th/0501065.

53. R. Lednicky, V.V. Lyuboshitz and V.L. Lyuboshitz, Phys. of Atomic Nuclei 61 (1998) 2050.

54. Yu.M. Sinyukov et al., Phys. Lett. 432B (1998) 248.

55. S. Pratt, Phys. Rev. Lett. 53 (1984) 1219; Phys. Rev. D 33 (1986) 1314.

56. Y. Hama and S. S. Padula, Phys. Rev. D 37 (1988) 3237.

57. A.N. Makhlin and Yu.M. Sinyukov, Z. Phys. C 39 (1988) 69.

58. U. Wiedemann and U. Heinz, Phys. Rep. 319 (1999) 145. 
59. T. Csörgö, Heavy Ion Phys. 15 (2002) 1.

60. M.I. Podgoretsky, Sov. J. Nucl. Phys. 37 (1983) 272.

61. G.F. Bertsch, P. Danielewicz and M. Herrmann, Phys. Rev. C 49 (1994) 442.

62. S. Pratt, in Quark Gluon Plasma 2 (ed. R.C. Hwa), World Scientific, Singapore, 1995, p.700.

63. S. Chapman, P. Scotto and U. Heinz, Phys. Rev. Lett. 74 (1995) 4400.

64. U.A. Wiedemann, Phys. Rev. C 57 (1998) 266.

65. T. Csörgő and S. Pratt, Proc. of the Workshop on Heavy Ion Physics, KFKI-199128/A, p.75.

66. S.S. Adler et al. (PHENIX), Phys. Rev. Lett. 93 (2004) 152302.

67. J. Adams et al. (STAR), Phys. Rev. C 71 (2005) 044906.

68. J. Adams et al. (STAR), Phys. Rev. Lett. 93 (2004) 012301.

69. S. Soff, S.A. Bass and A. Dumitru, Phys. Rev. Lett. 86 (2001) 3981.

70. U. Heinz and P.Kolb, Nucl. Phys. A 702 (2002) 269.

71. T. Csörgö and L.P. Csernai, Phys. Lett. 333B (1994) 494.

72. A. Dumitru and R.D. Pisarski, Nucl. Phys. A 698 (2002) 444.

73. Z.W. Lin, C.M. Ko and S. Pal, Phys. Rev. Lett. 89 (2002) 152301.

74. T. Humanic, Nucl. Phys. A 715 (2003) 641.

75. N. Borghini, arXiv:nucl-th/0509092.

76. P.F. Kolb and R. Rapp, Phys. Rev. C 67 (2003) 044903.

77. D. d'Enterria and D. Peressounko, arXiv:nucl-th/0503054

78. R. Rapp, Phys. Rev. C 66 (2002) 017901.

79. T. Hirano and K. Tsuda, Phys. Rev. C 66 (2002) 054905.

80. F. Grassi, Y. Hama and T. Kodama, Phys. Lett. 355B (1996) 9.

81. Yu.M. Sinyukov, S.V. Akkelin and Y. Hama, Phys. Rev. Lett. 89 (2002) 052301.

82. S.A. Bass and A. Dumitru, Phys. Rev. C 61 (2000) 064909.

83. D. Teaney, J. Lauret and E.V. Shuryak, arXiv:nucl-th/0110037.

84. Y. Hama, arXiv:hep-ph/0510096, these proceedings.

85. W. Florkowski, arXiv:nucl-th/0509039, these proceedings.

86. P. Csimandia, T. Csörgő and B. Lukács, Phys. Lett. 433B (1998) 21.

87. N. Amelin et al., arXiv:nucl-th/0507040.

88. M. Lisa et al. (E895), Nucl. Phys. A 698 (2002) 185.

89. F. Wang and S. Pratt, Phys. Rev. Lett. 83 (1999) 3138.

90. M. Gmitro et al., Czech. J. Phys. B 36 (1986) 1281.

91. S. Pislak et al., Phys. Rev. Lett. 87 (2001) 221801.

92. G. Colangelo, J. Gasser and H. Leutwyler, Phys. Lett. 488B (2000) 261.

93. S.V. Akkelin and Yu.M. Sinyukov, Z. Phys. C 72 (1996) 501.

94. T. Csörgő and A. Ster, arXiv:nucl-th/0207016.

95. M. Csanád et al., J. Phys. G: Nucl. Part. Phys. 30 (2004) S1079. 